Rhode Island College

Digital Commons @ RIC

\title{
Improvement of Patient Satisfaction Through Enhancement of RN Communication Skills: A Quality Improvement Project
}

Pamela A. Kusiak

Rhode Island College

Follow this and additional works at: https://digitalcommons.ric.edu/etd

Part of the Nursing Commons

\section{Recommended Citation}

Kusiak, Pamela A., "Improvement of Patient Satisfaction Through Enhancement of RN Communication Skills: A Quality Improvement Project" (2016). Master's Theses, Dissertations, Graduate Research and Major Papers Overview. 158.

https://digitalcommons.ric.edu/etd/158

This Major Paper is brought to you for free and open access by the Master's Theses, Dissertations, Graduate Research and Major Papers at Digital Commons @ RIC. It has been accepted for inclusion in Master's Theses, Dissertations, Graduate Research and Major Papers Overview by an authorized administrator of Digital Commons @ RIC. For more information, please contact digitalcommons@ric.edu. 


\title{
IMPROVEMENT OF PATIENT SATISFACTION THROUGH ENHANCEMENT OF RN COMMUNICATION SKILLS: A QUALITY IMPROVEMENT PROJECT
}

by

\author{
Pamela A Kusiak
}

A Major Paper Submitted in Partial Fulfillment

of the Requirements for the Degree of

Master of Science in Nursing

in

The School of Nursing

Rhode Island College

2016 


\begin{abstract}
Studies have shown that people with low health literacy understand health information less well, obtain preventative healthcare such as screenings for cancer less frequently and use expensive resources such as emergency rooms more often. The Agency for Healthcare Research and Quality (AHRQ), with guidance from Institute of Medicine (IOM) reports, developed quality indicators for individual institutions to demonstrate improvement. The Health Resources and Services Administration (HRSA, 2012) constructed online communication education modules for healthcare professionals including health literacy tools developed by the AHRQ. The purpose of this project was to examine the impact of RN communication education on patient satisfaction. A prepost intervention design was used. Nurses were provided a brief overview and instruction about the HRSA website, use of the online education modules and an explanation of target patient satisfaction scores. The domains studied included five target patient satisfaction questions regarding communication with nurses and communication about medications. Post intervention results (July-December 2015) demonstrate a marked increase in scores when compared to the six months prior to the introduction of the content (January-June 2015). All twenty-two respondents responded agreed or strongly agreed that they would recommend the Effective Communication for Healthcare Professionals 100 course. APRNs have an unprecedented opportunity to support research and education surrounding ethnic differences in communication and aspects of communication that may contribute to patient comprehension, adherence to follow-up care and patient satisfaction.
\end{abstract}




\section{Table of Contents}

Background/Statement of the Problem ...........................................................................

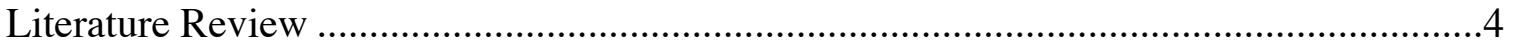

Theoretical Framework..................................................................................... 18

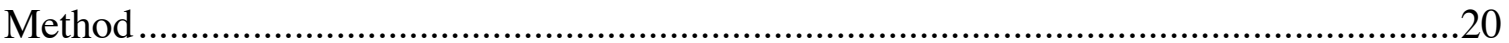

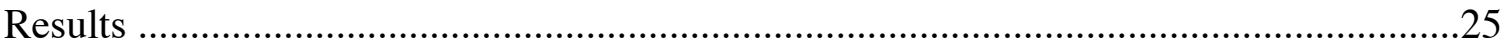

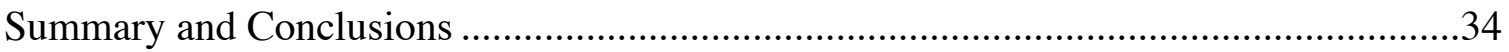

Recommendations and Implications for Advanced Nursing Practice ............................38

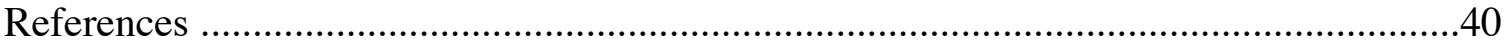

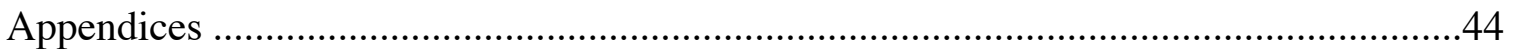


Improving Patient Satisfaction through Enhancement of RN Communication Skills:

A Quality Improvement Project

\section{Background/Statement of the Problem}

The Institute of Medicine's Committee on the Quality of Healthcare in America published a report in 2001 discussing the adverse relationship between the complexity of United States (US) healthcare advancements and decreased efficiency (Institute of Medicine [IOM], 2001). Within this report, six aims were developed to describe the core needs of the healthcare system as a whole. The first aim is safety, avoiding injuries to patients from the care that is intended to help them. The second aim, efficacy, relates to providing care that is evidence based. Patient centered care, the third aim, addresses that all patients deserve respect and should have their individual preferences, needs and values included in clinical decisions. The fourth and fifth aims are timeliness and efficiency, avoiding harmful delays and waste. Finally, the sixth aim describes equity, providing care that does not vary in in quality due to personal characteristics such as, gender race, geographic location and socioeconomic status. Achieving gains in these six categories would be beneficial in meeting needs of patients (IOM). The IOM report of 2001 contributed many of the changes in our healthcare system initiated by the Affordable Care Act. The overall goal was for patients to experience care that is safer, more reliable, more responsive to their needs, more integrated and more available (IOM, 2001).

The Agency for Healthcare Research and Quality (AHRQ), with guidance from the IOM report of 2001 and subsequent IOM reports, developed quality indicators for individual institutions to demonstrate improvement. Press Ganey Associates Incorporated, founded in 1985, began developing surveys that would measure patients' satisfaction as a means to improve performance. These surveys have been developed through the use of subjective experience data and objective research. Organizations that 
provide positive patient experiences also are more likely to have positive clinical and financial outcomes. In November 2012, Press Ganey launched the Institute for Innovation, a nonprofit research collaborative that provides a forum to better understand and act upon the patients' voice to advance the science of improving patient care and performance (Press Ganey, 2012). The Hospital Consumer Assessment of Healthcare Providers and Systems (HCAHPS) surveys are the first nationally standardized publicly reported surveys of patients' perceptions regarding the care experienced during hospitalization. Patients complete surveys composed of 27 questions in 18 core categories after discharge. Communication with hospital staff, nurses, and doctors is part of the core categories.

The first of the four strategies institutions can target to improve patient satisfaction involves communication, notably to communicate clearly and often (Rodek, 2012). As healthcare providers, it is not enough to provide the information needed to patients in a standardized form. It is necessary to individualize the explanations and confirm the individual patient understanding. This involves health literacy, which is defined as the degree to which individuals have the capacity to obtain, process, and understand basic health information and services needed to make appropriate health decisions (IOM, 2004).

A unified communication approach is necessary to address patients' ability to understand and participate in improving their own outcomes. This approach requires healthcare providers to assess the patient's health literacy, level of English proficiency, and develop an understanding of the patient's cultural influences to provide patient centered care. The US Department of Health and Human Service's Health Resources and Service Administration (HRSA) is the primary federal agency for improving access to healthcare by strengthening the healthcare workforce through education and information resources. The Health Resources and Services Administration developed free online communication education modules for healthcare providers in 2012, aptly named 
Effective Communication Tools for Healthcare Professionals 100. The basis of these modules is the development of a unified communication approach. Within the modules are the introduction to various assessment tools and communication strategies provided by the AHRQ. The purpose of this quality improvement project was to examine the impact of effective communication education for RNs on patient satisfaction.

Next, the review of the literature will be presented. 


\section{Literature Review}

The following literature was obtained from the use of PubMed, CINAHL and Medline data bases using key words and phrases that included patient satisfaction, patient communication, patient centered care, health literacy, effective communication, communication tools, quality communication, provider communication education, and teach back method. The search included literature published from November, 2001December, 2015.

\section{Patient-centered Care}

The Institute of Medicine's Committee on the Quality of Healthcare in America published a report in 2001 discussing the adverse relationship between the complexity of US healthcare advancements and decreased efficiency (IOM, 2001). Included in the IOM report were general guidelines for redesign to achieve the six aims for improvement. Care should be based on continuous healing relationships and customized according to patients' needs and values. The patient should be the source of control, provided with the necessary information and the opportunity to exercise the degree of control they choose over healthcare decisions that affect them. The health care system should be able to accommodate differences in patient preferences and encourage shared decision making (IOM). The IOM defined patient centered care as providing care that is respectful of and responsive to individual patient preferences, needs, and values, and ensuring that patient values guide all clinical decisions (IOM).

Patient centered care is a quality of personal, professional and organizational relationships (Epstein \& Street, 2011). There have been concerns that patient centered care has a focus on individual needs and may be at odds with an evidenced based practice approach, which tends to focus on populations. Evidenced based medicine must provide a good outcome in terms of what is meaningful and valuable to the individual patient. Patient centered care and evidenced based medicine both require the art of generalizations and the science of particulars. (Epstein \& Street, 2011). Evidence 
demonstrates that patient centered care improves disease outcomes and quality of life, and that it is critical to addressing racial, economic and social disparities in healthcare (Epstein, Fiscella, Lesser, \& Stange, 2010). Policies should assist healthcare professionals in acquiring and maintaining skills needed to support patient centered care. Organizations should strive to create a culture of patient centeredness (Epstein et al., 2010).

Patient centered care has been described in much of the literature as humanistic, with an emphasis on patient perspective and participation (Burman, Robinson, \& Hart, 2013). In contrast, evidence-based practice is considered a rationale-based approach (Burman et al.). Definitions of evidenced based practice used in nursing include patient preferences as a critical step in practice decisions and making changes in plans of care (Burman et al.). Four critical elements have been identified as successful in aligning patient centered care and evidenced based practice: health care redesign; decision support; empowered organizational culture; and informed and empowered nurses. In addition, productive interaction between informed patients and a prepared, proactive practice team is incorporated into successful comprehensive models of care (Burman et al.). In addition, patient engagement has become interchangeable or included in the overall definition of patient centered care. A guide to patient and family engagement was developed in collaboration with the AHRQ (Pelletier \& Stichler). The resulting definition highlights the importance of patient engagement as a central focus of patient centered care (Pelletier \& Stichler, 2014). An important component of providing patient centered care includes health literacy.

\section{Health Literacy}

The IOM Committee on Health Literacy released a report in 2004 titled Health Literacy: A Prescription to End Confusion. This report discussed that while causal relationships between low health literacy and poorer patient outcomes are yet to be determined, cumulative and consistent findings suggest a causal connection between the 
two. Studies have shown that people with low health literacy understand health information less well, get less preventative healthcare such as screenings for cancer, and use expensive resources such as emergency rooms more frequently (IOM, 2004). This report also suggested that the Department of Health and Human Services should take the lead on developing uniform standards for addressing health literacy and healthcare systems should develop and support demonstration programs to establish effective approaches to reduce the effects of low health literacy.

It is necessary to individualize the explanations and confirm the individuals' understanding. Health literacy is defined as the degree to which individuals have the capacity to obtain, process, and understand basic health information and services needed to make appropriate health decisions (IOM, 2004). The NIH Office of Communications and Public Liaison (OCPL) and its 27 component public affairs offices work to connect research with the public. This office serves as liaison to the Department of Health and Human Services (HHS) on a number of issues related to health literacy. Health literacy incorporates a range of abilities, including reading, comprehending, and analyzing information; decoding instructions, symbols, charts, and diagrams; weighing risks and benefits; and ultimately making decisions and taking action ("Clear Communication," 2015).

Studies indicate that patients have difficulty understanding health information that is communicated orally during the patient-clinician interaction. Patients retain about $50 \%$ percent of the information discussed. Studies show that those with limited health literacy are less likely to ask questions during a medical encounter, or understand medical terminology (IOM, 2004). Incorporating patient preferences into practice is challenging because of variations in patient preferences and decision making processes (Burman et al.). In addition, patients may not have enough time to reflect on their options and the evidence to come up with a definitive decision in a brief healthcare encounter (Burman et al.). Lack of knowledge by patients and different healthcare beliefs may be factors in the 
lack of alignment between patients and clinicians (Burman et al.). While it is known that ethnic differences in communication needs exist, little research has explored the aspects of communication that may contribute to patient comprehension, adherence to follow-up care, and satisfaction across ethnicity (Molina et al.2014).

A cross-sectional study of enrollment data from a randomized controlled trial of interventions to improve patient adherence to hypertension treatments was conducted by The Armstrong Institute for Safety and Quality, Johns Hopkins Healthcare Maryland in 2013. The objective was to examine how health literacy influences patients' interest in participating in healthcare visit communication and patient reported outcomes. The study participants were 41 primary care physicians and 275 of their patients. Measurements included the Rapid Estimate of Adult Literacy in Medicine (REALM) tool, patients' desire for involvement in decision making, trust and satisfaction. The REALM is a two to three minute reading cognition test that can be administered by a nurse and is used to measure the participant's ability to interpret health care terms and health related materials. A score of 18 or below indicates a third grade reading level, 19-44 is indicative of a fourth to six grade reading level, and a 61 or above indicates a ninth grade reading level. The adequate health literacy group included 173 patients, of which 92 or approximately 54\% had controlled blood pressure. The low literacy group included 102 patients of which 40 or approximately $40 \%$ had controlled blood pressure. Results suggested that while both groups were similarly interested in participating in medical decision making, a lower percentage of patients with lower versus adequate health literacy had controlled blood pressure. In addition, those with lower literacy asked fewer questions (Aboumatar, H. J., Carson, K. A., Beach, M. C., Rotor, D. L., \& Cooper, L. A., 2013).

A pre-post intervention study was conducted by a surgeon group from the Maricopa Medical Center in Phoenix Arizona (Komenaka et al., 2014). The purpose was to determine the feasibility of a health literacy assessment and its' effect on patient 
satisfaction. Every patient seen in a breast surgery clinic during a two year period was asked to undergo a health literacy assessment with the Newest Vital Sign (NVS) tool as a part of the routine history and physical examination. The NVS tool consists of a nutrition label design similar to that of most food labels here in the US. The assessment involves asking the patient five questions regarding the label content, and a sixth qualifier question if needed. The patient receives a point for each correct answer. Scores of 0-1 indicate a high likelihood of limited health literacy, scores of 2-3 indicate possibility of limited health literacy and 4-6 indicate adequate health literacy. Approximately 2025 patients seen in the two year period were eligible and only one refused to participate. The majority $(\mathrm{n}=1,229 ; 61 \%)$ of the patients had a NVS score of $0-1$, indicating a high likelihood of low health literacy. There were 405 patients (20\%) that scored 2-3, indicating a possibility of low health literacy and 391 (19\%) scored in the adequate health literacy range of 4-6. In 2009, the year prior to routine health literacy assessments, the mean satisfaction score was 3.7. In the first year of the NVS being conducted with the routine health assessments, the mean satisfaction score improved slightly to 3.8 ; during the second year, the mean satisfaction score was reported at 4.1. These results suggest that health literacy assessment is feasible in surgical practice, and also that improved health literacy can result in increased patient satisfaction (Komenaka et al.).

\section{Patient Satisfaction}

Patient satisfaction is the health care recipient's reaction to aspects of his or her service experience. If a patient's perception of their hospital experience meets or exceeds the expectation, there will be a corresponding degree of satisfaction (Torcson, 2005). Patient satisfaction refers to what patients think about their treatment, while evaluation of the patients' experience focuses on establishing protocols for care coordination, communication with caregivers and staff responsiveness (Maat, 2013).

Patient perceptions of care can be measured directly from patient satisfaction surveys. Patient perception of quality is assessed through dimensions of what is 
personally valued, and often they do not distinguish between the provider of the service and the service received (Torcson). The surveys ask patients about important aspects of their visit, interaction with health care professionals and their hospital experience. Patient satisfaction belongs to the service dimension as opposed to the technical dimension of quality of care. Most patients report few problems related to technical quality of care in hospitals and moreover do not feel qualified to judge technical quality and therefore assume technical competence. Being treated with respect and dignity and involvement in treatment decisions are intangible issues of patient satisfaction that are paramount issues for patients (Torcson). Organizational cultures must value patient preferences through support, rewards and recognition, strategic planning, and learning environments. Nurses should be informed and empowered to lead efforts to redesign the healthcare system so that patients make their own decisions based on evidenced based options (Burman et al., 2013). The Hospital Consumer Assessment of Healthcare Providers and Systems (HCAHPS) surveys are the first nationally standardized, publicly reported surveys of patients' perceptions regarding the care experienced during hospitalization. Patients complete surveys composed of 27 questions in 18 core categories after discharge. Communication with hospital staff, nurses, and doctors are part of the core categories (Press Ganey, 2012).

The Massachusetts Health Quality Partnership (MHQP) designed a program to measure and disseminate comparative quality information about hospital inpatient experiences as part of a statewide patient survey project (Rogers \& Smith, 1999). As many as one quarter of 13,000 patients discharged from 51 Massachusetts hospitals reported problems with patient satisfaction issues such as the involvement of families in care, communication and coordination of care, and the transition from hospital to home. Expectations of patients as hospital customers rise from past experiences of their own or of others, as well as from current needs and unique internal preferences that form the basis of a value system (Torcson). 
Hospitals have experienced increased patient satisfaction levels, decreased costs, and lengths of stay after implementing patient centered philosophies (Pelletier \& Stichler, 2014). Studies have demonstrated that a patient centered approach to care in both inpatient and out-patient services has resulted in statistically significant changes in the perceptions of patients in finding common ground, improved emotional and physical health status, increased efficiency of care and greater self-efficacy (Pelletier \& Stichler).

In an exploratory, qualitative study conducted between February and September of 2012, women were recruited from two mobile mammography services affiliated with the National Breast and Cervical Cancer Early Detection Program (NBCCEDP) grantee program. The purpose of this study was to identify patients' communication needs and experiences with follow-up care among Latina and non-Latina white women who received an abnormal mammogram. Despite barriers to comprehension, Latina respondents did not discuss the provision of clear information as a requisite for a positive follow-up appointment experience. Instead, they highlighted their need for empathic care providers who demonstrated patience and willingness to spend time with them, through their tone of voice and body language. Recommendations concerning how to communicate to patients often included a need for empathy with Latinas, as indicated by respondents' suggestions. The authors suggested that to improve patient satisfaction and follow-up care among Latinas educational programs are necessary to counsel healthcare professionals with regard to language, health literacy, and empathetic communication needs (Molina et al., 2014).

Through interpersonal aspects of professional nursing care, nurses can establish relationships that support patient engagement and activation, which can lead to improved outcomes (Pelletier \& Stichler). Nurse leaders must ensure that nurses and other healthcare providers have the competencies necessary to ensure patient centered care (Pelletier \& Stichler).

\section{Effective Health Communication}


A unified communication approach is necessary to address a patient's ability to understand and participate in improving his/her own outcomes. This approach requires healthcare providers to assess the patient's health literacy, level of English proficiency, and develop an understanding of the patient's cultural influences to provide patient centered care (HRSA, 2012).

Patients' ability to follow instructions is heavily influenced by providers' communication styles. In addition, health conversations often take place during a period of high anxiety for the patient, limiting the patient's ability to retain information. Healthcare providers must adapt their approach, dependent on the assessment of patients' responses (HRSA, 2012). As healthcare providers, it is not enough to provide the information needed to patients in a standardized form. It is necessary to individualize the explanations and confirm the individual patient understanding (HRSA). Health professionals today are a multigenerational, diverse working group with individual psychosocial, spiritual and life experience characteristics of their own that shape their practice (Duffy, 2013). The ability to develop a compassionate, therapeutic relationship with patients and engage them as partners, is critical as healthcare will require patients to be fully informed and active participants in self-care management (Pelletier \& Stichler). In addition, many are caring for the most diverse, acute and chronically ill population this nation has ever seen (Duffy).

In the acute hospital setting, professional nurses supervise unlicensed personnel, provide care 24/7, chase down equipment and supplies, coordinate healthcare teams, and participate in shared governance councils and performance improvement projects (Duffy). Meanwhile, economic constraints and cost containment strategies challenge nurses in a 'do more with less' environment (Duffy). "Health professionals today are frequently working at the interface between the efficiency needs of the health system and the human caring needs of patients and families" (Duffy, p. 61). 
In 2008, The Robert Wood Johnson foundation (RWJF) and the IOM launched a two year initiative to respond to the need to transform the nursing profession. The US has the opportunity to transform the healthcare system and nurses can and should play a fundamental role (IOM, 2010). The IOM report that followed titled The Future of Nursing: Leading Change, Advancing Health made recommendations for the future of nursing, most aimed at advancing education and enhancement of academic programs. The core principles of a unified communication approach, health literacy, cultural competency, and assessment of level of English proficiency are being added to the nursing curriculum at colleges and universities. However, research surrounding enhancing communication skills of nurses currently working in the acute setting is limited.

A prospective cohort study was conducted by White et al. (2013) at the University of California Medical Center, San Francisco. The purpose of the study was to determine if hospitalized heart failure patients educated with the teach-back method retained selfcare educational information and if this was associated with lower readmission rates. The concept underpinning teach-back education involves asking patients to restate information that has been presented to them. Teach-back is an interactive process rather than simply providing information. This technique allows the educator to check for lapses in recall and understanding, reinforce and tailor messages, and engage in an open dialogue with patients. (White et al.).

The study included 278 patients over the age of 65 , hospitalized with a diagnosis of heart failure, and was conducted over a 13 month period. Patients were educated by two heart failure registered nurse coordinators during hospitalization for heart failure. The two nurses who provided the education were introduced to the teach-back method of education during a course offered through the Institute for Healthcare Improvement (IHI) an independent nonprofit organization assisting healthcare systems to improve patient care by integrating research and education. Data on the ability to recall information after 
a seven day period post hospital discharge were collected. The results of this study demonstrated that the teach-back method was an effective tool to educate and assess learning. Approximately $84 \%(n=233)$ of patients correctly answered three of four teachback questions while hospitalized and $77.1 \%(\mathrm{n}=145)$ answered correctly during followup. One challenge of the teach-back method is that it is difficult to control the fidelity of procedures because of the interactive and open nature of the method. The nurse using the teach-back method must also assess retention of learning and, when necessary, provide supplemental education until learning is achieved, leaving the potential for bias (White et al.). While there was no significant improvement for overall hospital re-admission rates, there was a trend toward decreasing the number of patients admitted for heart failure.

Ethnic differences in communication needs and experiences with healthcare staff and providers may contribute to delays as well as satisfaction with care. To improve patient satisfaction and adherence to follow-up care, educational programs are necessary to counsel health-care professionals with regard to language, health literacy, and empathetic communication needs (Molina et al.). First, understanding culture helps us to understand how others interpret their environment. We know that culture shapes how people see their world and how they function within that world. Culture shapes personal and group values and attitudes, including perceptions about what works and what doesn't work, what is helpful and what is not, what makes sense and what does not ("Cultural competency and diversity", 2014). Also, understanding culture will assist clinicians in avoiding stereotypes and biases that can undermine their efforts. It promotes a focus on the positive characteristics of a particular group, and reflects an appreciation of cultural differences. Finally, culture plays a complex role in the development of health and human service delivery programs ("Cultural competency and diversity").

Communication provides an opportunity for persons of different cultures to learn from each other. It is important to build skills that enhance communication. When professionals are culturally competent, they establish positive helping relationships, 
engage the client, and improve the quality of services they provide. Cultural competence is rooted in respect, validation and openness towards someone with different social and cultural perceptions and expectations than your own ("Cultural competency and diversity").

\section{Effective Communication Tools 100: A Unified Communication Approach}

The HRSA in 2012 developed free online communication education modules for healthcare providers, aptly named Effective Communication Tools 100, using a unified communication approach. Within the modules are the introduction to various assessment tools and communication strategies provided by the AHRQ. Module 1 provides an in depth overview of effective health communication, the direct benefit on medical outcomes and definition of a unified communication approach. This module directly correlates to mutual problem solving and focuses on the importance of communication in maintaining patient centered care. Effective health communication contributes to lower rates of anxiety, pain and psychological distress in patients and thus increased trust between patients and providers, essential in the application of attentive reassurance or conveying reliability to the patient. Modules 2-4 examine health literacy, cultural competency and limited English proficiency. These modules review red flags and assessment tools to use in assessing a patient's learning needs, while providing a safe environment for the patient to communicate in an encouraging manner. Some of the techniques in module 2 include conducting a Newest Vital Sign Assessment (NVS) and Rapid Estimate of Adult Literacy in Medicine (REALM) in assessing health literacy. Module 3 focuses on cultural competency essential in the QCM factor, appreciation of unique meanings. This factor requires the provider to incorporate and or understand the patient's context or world-view. Module 4 covers limited English proficiency, appropriate techniques for use of interpreters and translators and assessment techniques. 
Module 5 concludes the course as a capstone activity that allows the learner to apply the unified communication approach in an interactive scenario. If health-care providers are to improve patient satisfaction, effective communication and understanding the patients' individual educational needs and influences are necessary.

Next, the framework that guided this study will be presented. 


\section{Theoretical Framework}

The Quality-Caring Model (QCM) by Joanne R. Duffy was chosen to guide the development of this project. This nursing model was developed with the patient and family, healthcare team and professional nurse centered on caring relationships. The major concepts of the QCM are humans in relationship, relationship-centered professional encounters, feeling "cared for" and self-advancing systems (Duffy, 2013). If healthcare providers establish relationship based encounters including patient and family, services are delivered more effectively. These collaborative relationships can be instrumental in gaining specific goals including patient satisfaction. When relationships are grounded in caring factors, a human connection occurs that is transpersonal and more than the individuals alone (Duffy).

Specific factors, namely mutual problem solving, attentive reassurance, human respect, encouraging manner, healing environment, appreciation of unique meanings, affiliation needs, and basic human needs, have been used as guides for understanding and practicing the QCM (Duffy). These eight caring factors are applied throughout the five modules of Effective Communication 100. Cultivating relational aspects of professional practice requires interaction, knowledge, and skills. Patients' perspectives of their healthcare experiences are essential to providing clinicians with information on how care processes affect responses to illness, and are now considered a unique and independent measure of quality (Duffy).

The QCM model differentiates how the term therapeutic implies healing whereas the term relationship denotes an interaction, bond and or partnership. This partnership factor is not limited to the patient provider context. It may be among peers, between disciplines or between the employee and the organization's leadership. Registered nurses (RNs), like many healthcare professionals are faced with adapting to economic constraints and cost containment strategies as our healthcare system evolves. Health 
professionals today are balancing the efficiency needs of the system and the human needs of patients and families (Duffy). The overarching goal in choosing the QCM model to guide this project was to support relationship based professional encounters, and to support RNs challenged with caring for the most diverse, acute and chronically ill patients.

The methodology for this project will be presented next. 


\section{Method}

\section{Purpose}

The purpose of this project was to examine the impact of providing staff RNs effective communication education modules provided by HRSA on aggregate post hospitalization patient satisfaction scores (HCAHPS).

\section{Design}

The project employed a pre-post intervention design.

\section{Sample and Site}

The sample included staff nurses employed on a 30 bed medical-surgical nursing unit at a 247 bed acute care hospital. All staff nurses were eligible to participate; there were no exclusion criteria. During the study period, nurses from the sister unit were floated to the study unit on a regular basis. Since these nurses cared for patients that would be receiving the satisfaction survey, these nurses were included in the opportunity to complete the modules. It is important to note that the study unit census fluctuated during the initiation of the project. Thus staffing was influenced by a greater number of sister unit staff working on the study unit and a larger number of staff nurses included. However, actual study results are reported only for the identified study unit.

\section{Procedures}

First, the project was approved by the Nurse Executive Committee at The Miriam Hospital and then submitted to the IRB at Lifespan for review as a quality improvement project.

An informational email was sent to staff nurses two weeks in advance of planned introductory meetings. The student investigator met with staff nurses on the study unit on various dates and times as determined in collaboration with the nurse manager and according to staff availability. The intent of these sessions was to introduce the nurses to the HRSA site and to provide background on the purpose of the project. Nurses were 
provided a brief overview and instruction about the HRSA website, how to access and use the Effective Communication 100 modules and an explanation of target HCAHP scores. The sessions were interactive, allowing for questions and fostered a teamwork approach. Contact information for the researcher was provided in the event that staff nurses had questions or needed assistance.

Participants were reminded that their participation at the informational session was optional, but that completion of the five training modules was mandated by the nurse manager. Participants were paid at the individuals' hourly rate for four hours of training time to complete the five modules. As part of completion of the modules, participants also responded to a survey provided by HRSA to obtain a certificate of completion. The certificate was returned to the nurse manager to verify completion of the mandated training. An anonymous evaluation survey (Appendix A) regarding the content of the modules and ease of use was distributed to participants when they turned in the certificate of completion.

\section{Intervention}

The US Department of Health and Human Service's, Health Resources and Services Administration (HRSA) is the primary federal agency for improving access to healthcare by strengthening the healthcare workforce. The Health Resources and Services Administration developed free online communication education modules for healthcare providers in 2012, aptly named Effective Communication Tools for Healthcare Professionals 100, using a unified communication approach. Within the five modules are the introduction to various assessment tools and communication strategies provided by the AHRQ (Table 1).

\section{Table 1}

Effective Communication Tools for Healthcare Professionals 100

\begin{tabular}{ll} 
Module $\quad$ Content & Time to complete \\
\hline
\end{tabular}




\begin{tabular}{lll}
\hline Module 1 & Overview effective & 45-60 minutes \\
& health communication & \\
Module 2 & Health Literacy & 60 minutes \\
Module 3 & Cultural Competency & $30-40$ minutes \\
Module 4 & Level of English & $30-40$ minutes \\
& Proficiency & \\
Module 5 & Capstone application & $10-20$ minutes \\
& activity
\end{tabular}

Testing provided at the end of each module, only required in module 5 to obtain certificate.

\section{Measurement}

Press Ganey Associates Incorporated founded in 1985, developed surveys to measure patients' satisfaction as a means to improve performance. The surveys were developed through the use of subjective experience data and objective research. The Hospital Consumer Assessment of Healthcare Providers and Systems (HCAHPS) surveys are the first nationally standardized publicly reported surveys of patients' perceptions regarding the care experienced during hospitalization. The surveys are distributed to all 
medical-surgical patients, inpatient and observation status. Patients complete surveys composed of 27 questions in 18 core categories after discharge. The survey also includes four screener questions and seven demographic items, which are used for adjusting the mix of patients across hospitals and for analytical purposes. The survey contains a total of three questions regarding communication with nurses and two regarding communication about medications. In addition, hospitals/survey vendors must submit a Participation Form to the HCAHPS Project Team for approval prior to the administration There are four approved modes of administration for the HCAHPS Hospital Survey: 1) mail only; 2) telephone only; 3) mixed (mail followed by telephone); and 4) active interactive voice response (IVR). Communication with hospital staff, nurses, and doctors is part of the core categories.

Patient satisfaction data related to aggregate patient satisfaction scores were collected. Pre and post unit-based, aggregate HCAHPS summary reports were compared six months prior to and six months after introduction of the HRSA modules. Results are presented in three month or quarterly intervals. The focus of this project was results from domains regarding communication with nurses and communication about medications, represented by the following questions:

- Nurses treat you with courtesy and respect;

- Nurses listen carefully to you;

- Nurses explain in a way you can understand;

- You were told what new medicine was for; and Staff described medicine side effects.

Results were accessed by the unit manager through an on-line secured access Press Ganey portal. Results were organized by discharge date, and included only the Top Box responses (Always).

Next, the survey results will be presented. 


\section{Results}

A total of 25 staff nurses attended informational sessions and 16 completed the educational modules. The sister unit included 36 staff nurses that attended informational sessions, and 17 completed the modules. A total of 22 module evaluation surveys were completed and returned. Two nurses completed the modules prior to the in-services, seven nurses completed them in August, nine in September, fourteen in October and one more by November 2015 .

\section{HCAHPS Scores}

The following graphs illustrate responses in the Communication with Nurses and Communication about Medications domains from discharged patients. Six months during and post intervention (July-December 2015) retrieved from Press Ganey improvement portal are displayed and compared to pre intervention period from (January-June 2015).

Figure 1 on the next page illustrates the results of the overall domain Communication with Nurses, which includes all three of the target responses in this domain. 


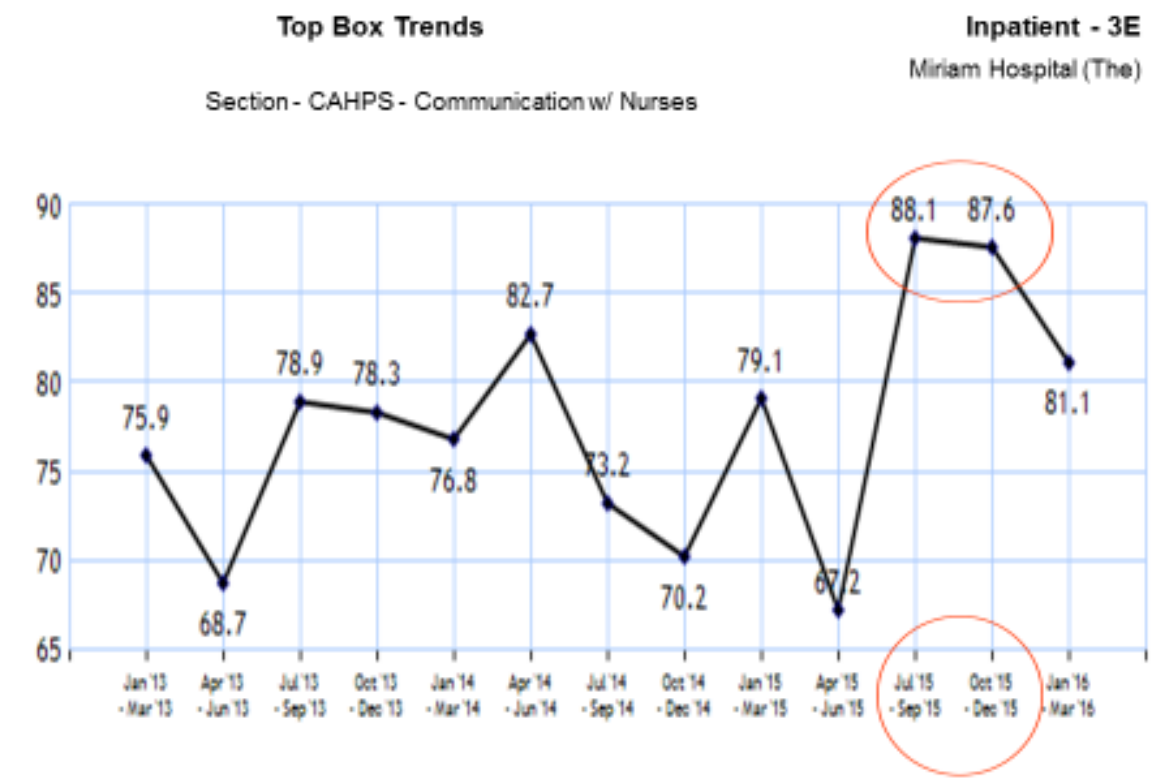

Displayed by Discharge Date

Figure 1. Overall scores: Communication with nurses domain

The first HRSA module in-services started in late July of 2015. Though not all completion certificates were returned during the first quarter, post intervention results (July-December 2015; represented in two quarters) demonstrate a marked increase in scores when compared to the six months prior to the introduction of the content (JanuaryJune 2015). Note that the next point on the graph (January-March 2016) appears as a downward trend; however, these results remain incomplete and should not be used for comparison.

Figure 2 illustrates the results for the individual question "Nurses treat you with courtesy and respect”. 


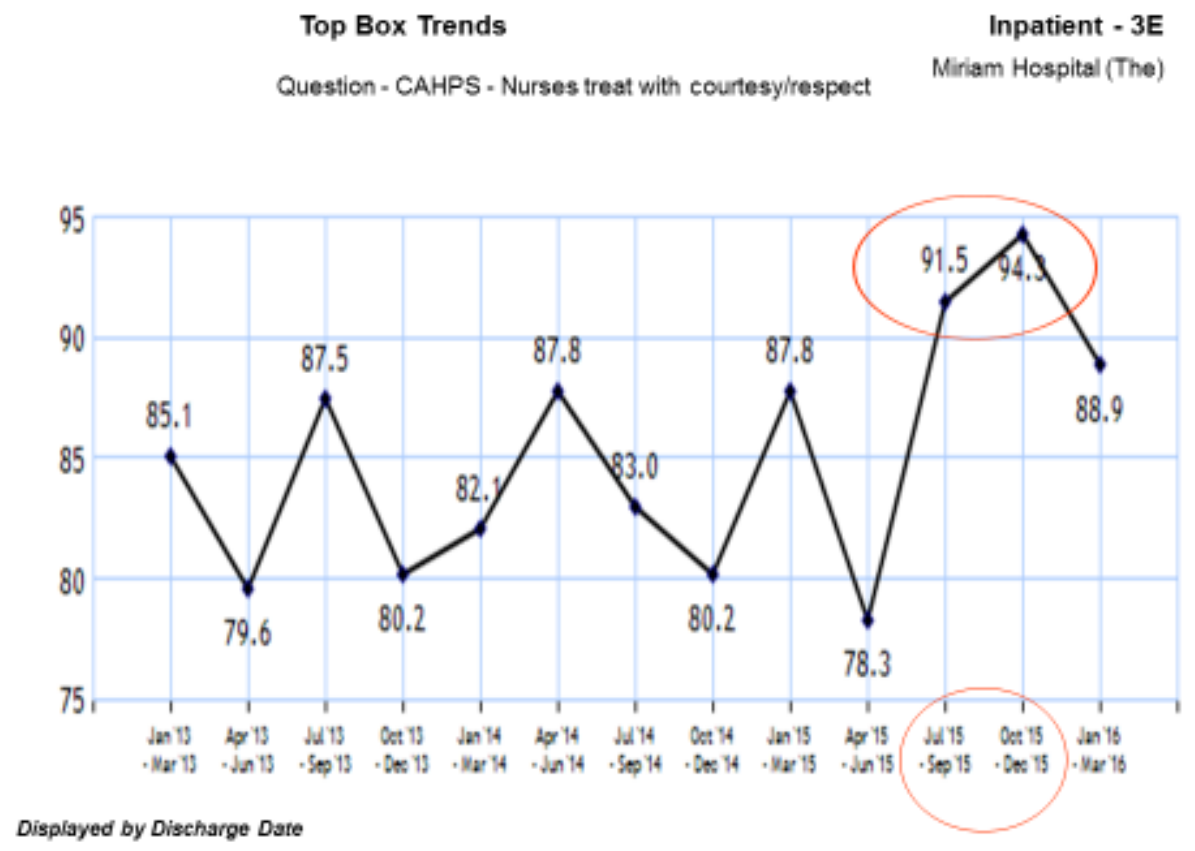

Figure 2. Individual question responses: "Nurses treat you with courtesy and respect"

For the individual question Nurses treat you with courtesy and respect, scores improved for the six months during and post introduction of the HRSA modules (JulyDecember 2015) as compared to the pre-intervention period from January-June 2015. The next point on the graph (January-March 2016) appears as a downward trend; however, these results remain incomplete and should not be used for comparison.

Figure 3 illustrates the results for the individual question "Nurses listen carefully to you”. 


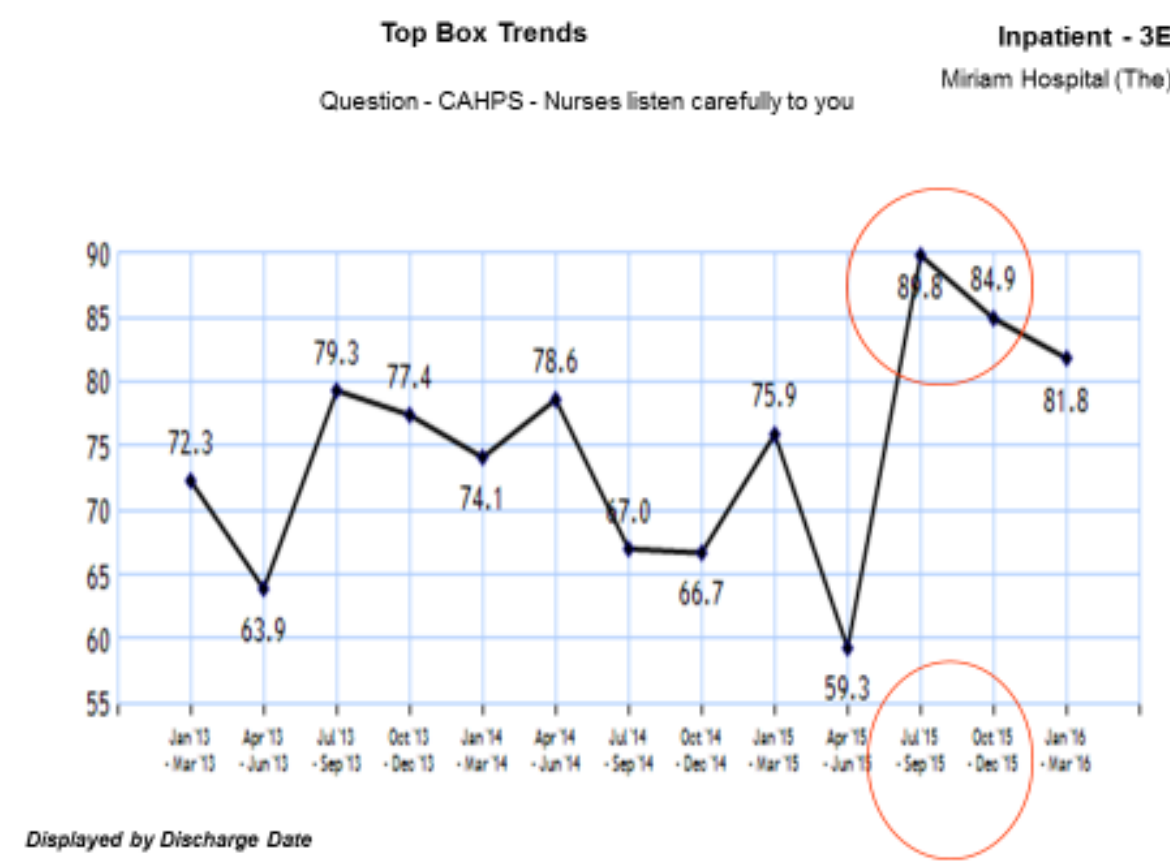

Figure 3. Individual question responses: "Nurses listen carefully to you"

For the individual question "Nurses listen carefully to you" scores improved during and post introduction of the HRSA modules (July- December 2015) as compared to the pre-intervention scores (January-June 2015). The next point on the graph (JanuaryMarch 2016) appears as a downward trend; however, these results remain incomplete and should not be used for comparison.

Figure 4 illustrates responses to the individual question Nurses explain in a way you can understand. 
Question - CAHPS - Nurses explain in way you understand

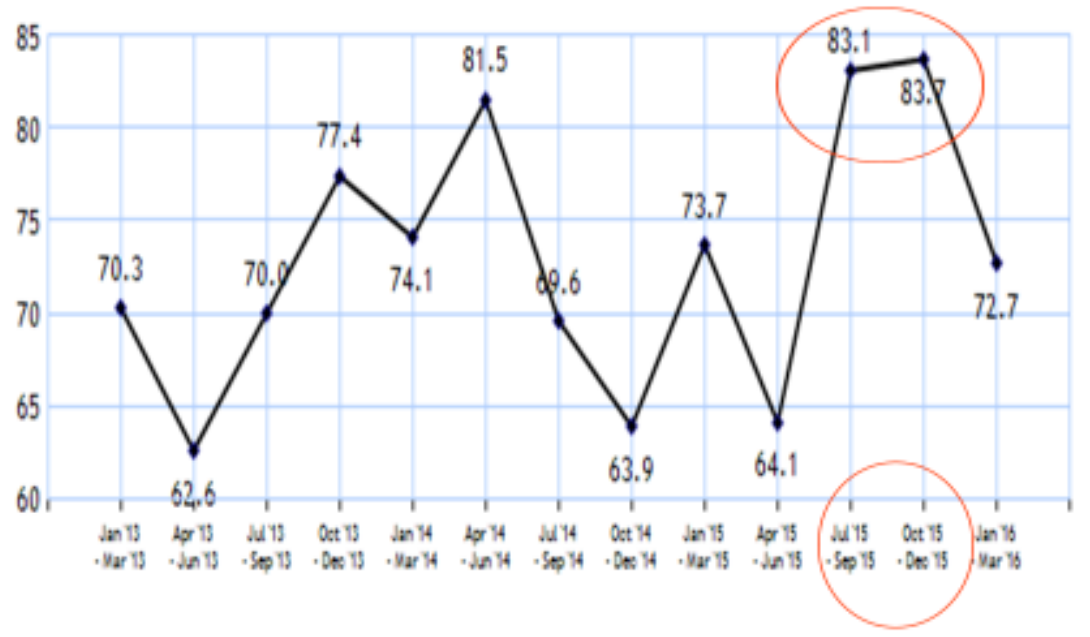

Displayed by Discharge Date

Figure 4. Individual question responses: "Nurses explain in a way you understand"

The third and final question of the communication with Nurses domain, "Nurses explain in a way you understand" also showed improvement. Scores for this question in the six months during and post introduction of the HRSA modules (July-December 2015) demonstrate a sustained improvement when compared to pre-intervention period (January to June 2015). The next point on the graph (January-March 2016) appears as a downward trend; however, these results remain incomplete and should not be used for comparison.

Next in Figure 5 are the results for the averaged domain Communication about Medications. 


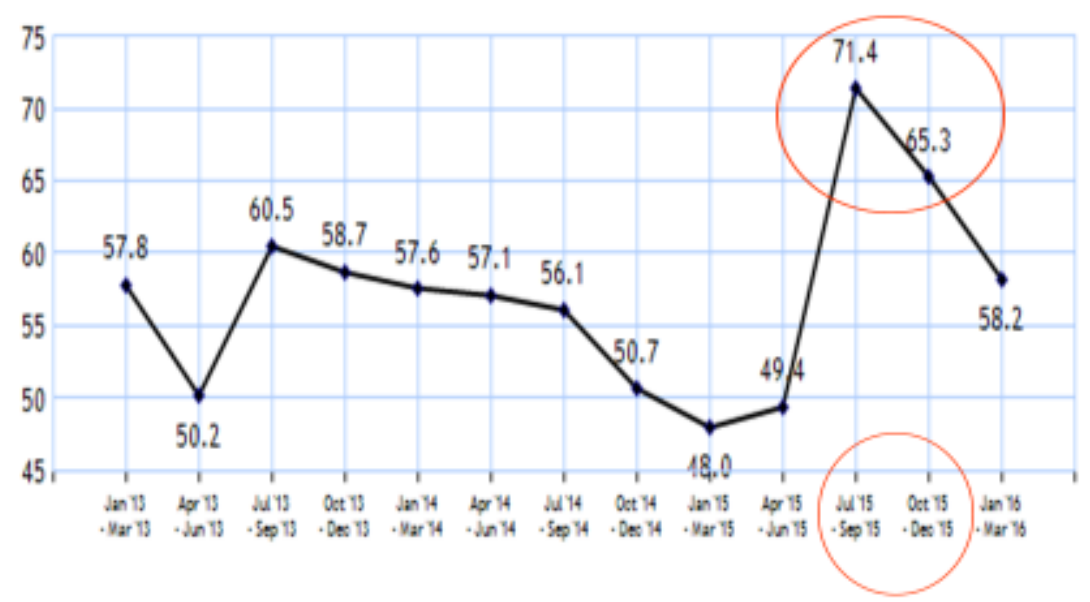

Displayed by Discharge Date

Figure 5. Scores overall: Communication about Medications domain

For the overall domain that included both target questions regarding Communication About Medications there was a marked increase from July-December 2015 during and post introduction of the HRSA modules when compared with the preintervention period (January to June 2015). The next point on the graph (January-March 2016) appears as a downward trend; however, these results remain incomplete and should not be used for comparison.

Next in Figure 6 are the results for the individual question Staff tell you what medication was for. 


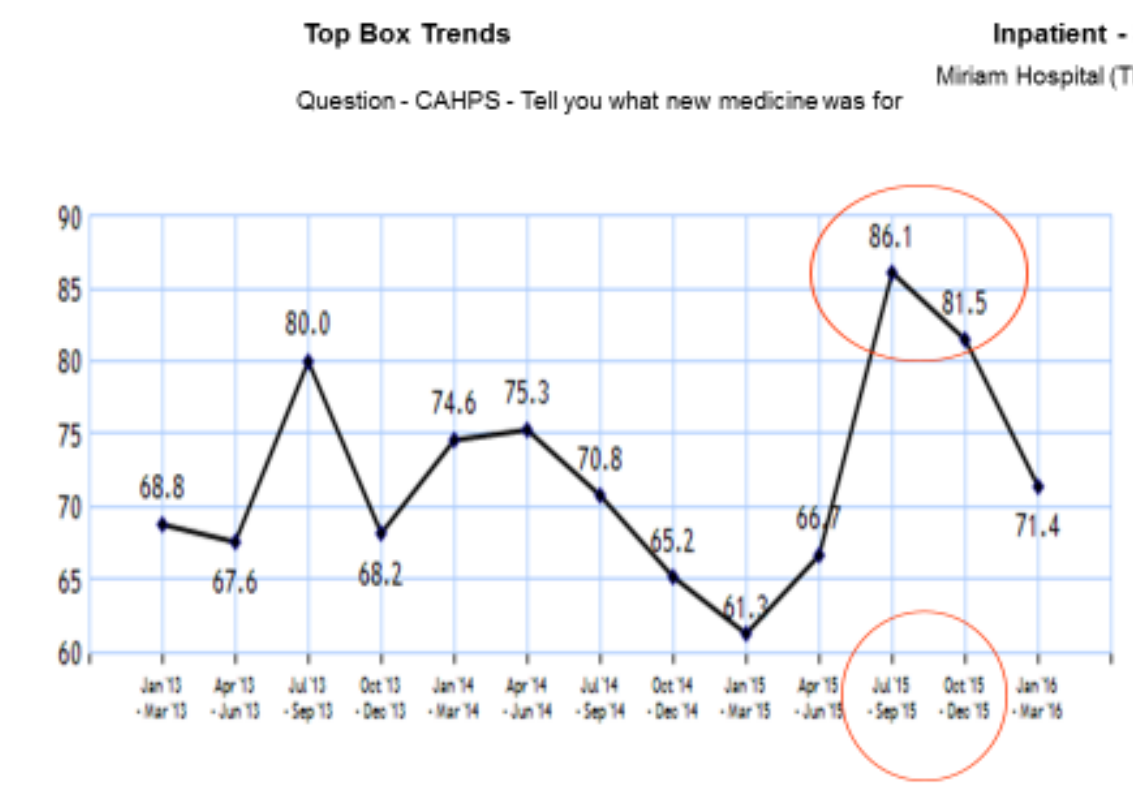

Displayed by Discharge Date

Figure 6. Individual question: "Staff tell you what medication was for"

For the individual question "Staff tell you what the medication is for" scores improved during and post introduction of the HRSA modules (July- December 2015). The next point on the graph (January-March 2016) appears as a downward trend; however, these results remain incomplete and should not be used for comparison. Illustrated in Figure 7 are the results for the individual question Staff explain medication side effects. 
Question - CAHPS - Staff describe medicine side effect

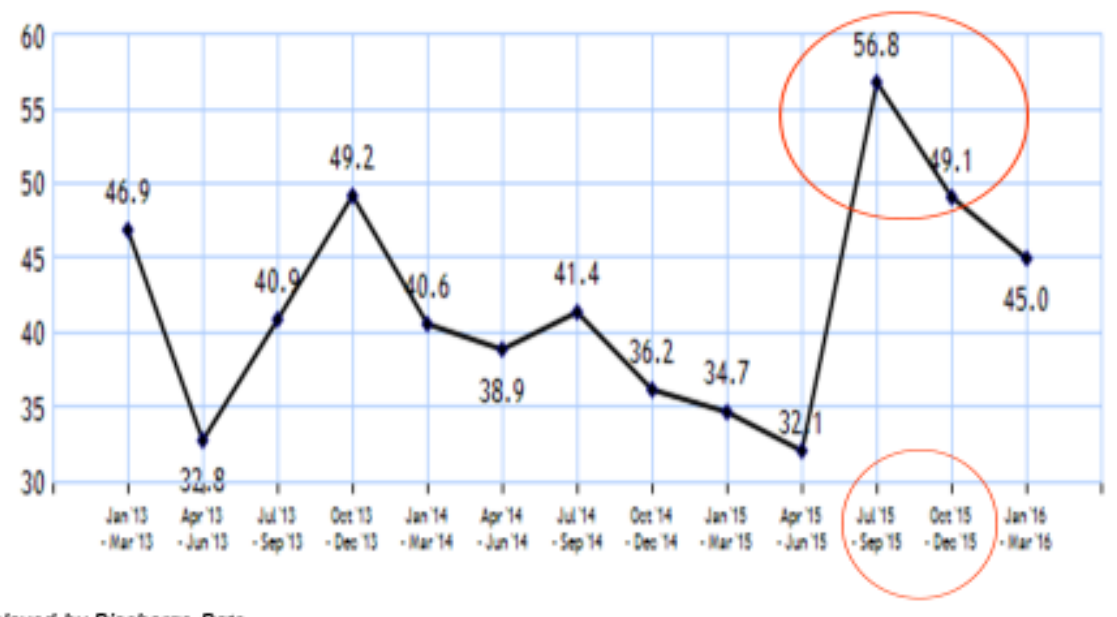

Displayed by Discharge Date

Figure 7. Individual question: "Staff describe medicine side effect"

For the individual question "Staff describe side effects of medicine" scores improved during and post introduction of the HRSA modules (July-December 2015). The next point on the graph (January-March 2016) appears as a downward trend; however, these results remain incomplete and should not be used for comparison.

\section{Evaluation}

Twenty two anonymous evaluation surveys (Appendix A) completed by the RN participants from both units were returned. Figure 8 illustrates the responses of the participants. 


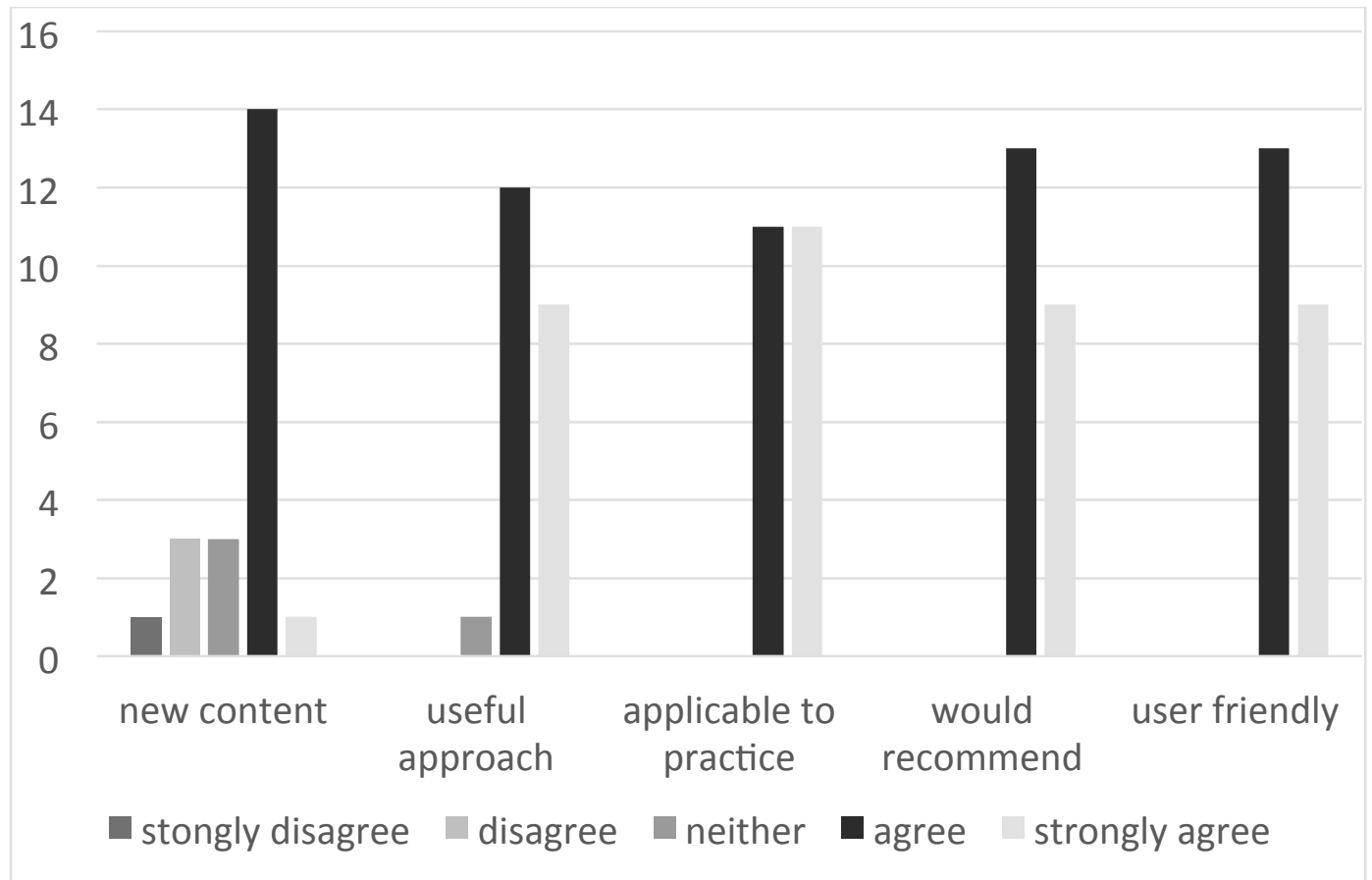

Figure 8. RN survey regarding HRSA modules

Fifteen out of the 22 respondents (68\%) agreed or strongly agreed that the modules provided content that was new to them. Twenty one (95\%) responded agreed or strongly agreed that the Unified Communication Approach was a useful way to communicate with their patients. All twenty-two respondents agreed or strongly agreed that the content in the modules was applicable to their practice. All twenty-two respondents responded agreed or strongly agreed that they would recommend the Effective Communications for Healthcare Providers course and that the modules were easy to use.

Next, summary and conclusions will be presented and discussed. 


\section{Summary and Conclusions}

The Health Resources and Services Administration (HRSA) developed free online communication education modules for healthcare providers in 2012, aptly named Effective Communication Tools 100, using a unified communication approach. Within the five modules are the introduction to various assessment tools and communication strategies provided by the AHRQ. The Hospital Consumer Assessment of Healthcare Providers and Systems (HCAHPS) surveys are the first nationally standardized publicly reported surveys of patients' perceptions regarding the care experienced during hospitalization. This quality improvement project compared patient satisfaction scores (HCAHPS) six months pre and post introduction of the HRSA modules to staff RNs.

This project took place in a Magnet designated hospital with a strong professional development culture supported by nursing leadership. Development of this project was guided by the "Quality-Caring Model” (QCM) which is a relationship-centered approach that had been incorporated into the hospital's professional nursing model. In general, staff nurses were familiar with the model and the focus on "healing-relationships". The major concepts of the QCM are humans in relationship, relationship-centered professional encounters, feeling "cared for" and self-advancing systems (Duffy, 2013).

The sample included staff nurses on a 30 bed medical-surgical nursing unit at a 247 bed acute care hospital. The student investigator met with staff nurses on the study unit to introduce the nurses to the HRSA site and to provide background on the purpose of the project. Due to shared staffing practices, staff on a sister unit were also included in the training but data compared was from the study unit only. Sixty-one staff nurses attended informational sessions and 33 completed the assigned modules.

The focus of this project was on the HCAHPS scores from domains regarding communication with nurses and communication about medications. Pre and post unitbased, aggregate HCAHPS summary reports were compared six months prior to (January-June 2015) and six months after introduction of the HRSA modules (July- 
December 2015). Though not all completion certificates were returned during the first quarter, post intervention results (July-December 2015; represented in two quarters) demonstrated a marked increase in scores when compared to the six months prior to the introduction of the content (January-June 2015) in both domains.

The communication with nurses domain included responses to three questions: Nurses treat you with courtesy and respect; Nurses explain in a way you can understand; and Nurses listen carefully to you. For the overall domain that included these three target questions, post intervention results (July-December 2015) demonstrated a marked increase in scores when compared to the six months prior to the introduction of the content (January-June 2015). The communication about medications domain included two questions, you were told what new medicine was for; and Staff described medicine side effects. For the overall domain that included both target questions regarding communication about medications, there was a marked increase from July-December 2015 during and post introduction of the HRSA modules when compared with the preintervention period (January to June 2015).

Twenty-two of the nurses completed an optional anonymous evaluation survey regarding the content of the modules and ease of use. Fifteen of the 22 respondents $(68 \%)$ agreed or strongly agreed that the modules provided content that was new to them. Twenty one (95\%) responded agreed or strongly agreed that the Unified Communication Approach was a useful way to communicate with their patients. All 22 respondents responded agreed or strongly agreed that they would recommend the Effective Communications for Healthcare Providers course and that the modules were easy to use.

Due to the measure used (aggregate patient satisfaction scores) and the limited time frame of comparison, the extent which the HRSA modules impacted nurse to patient communication remains unclear. This study was conducted over one year allowing for six months each for pre and post intervention comparison. The study unit included 25 of the nurses that attended the informational sessions and of those $16(64 \%)$ completed the 
HRSA modules. During initiation of this project, the study unit was undergoing renovation and had frequent fluctuations in patient census and the number of returned patient surveys. In addition, staff informational sessions were extended over several weeks to reach every staff member and not all the nurses completed the modules in the first quarter. Staff turned in module completion certificates over a three month period extending into the second quarter post intervention. Staff did express enthusiasm to be part of a quality improvement project, but had difficulty finding time to schedule in completion of the modules as training time during their regular work hours. Further evaluation and measures to sustain will need to be implemented.

Organizations that provide positive patient experiences also are more likely to have positive clinical and financial outcomes. "Health professionals today are frequently working at the interface between the efficiency needs of the health system and the human caring needs of patients and families" (Duffy, p. 61). Organizational cultures must value patient preferences through support, rewards and recognition, strategic planning, and learning environments. Nurses should be informed and empowered to lead efforts to redesign the healthcare system so that patients make their own decisions based on evidenced based options (Burman et al.). The health care system should be able to accommodate differences in patient preferences and encourage shared decision making (IOM, 2004). Being treated with respect and dignity and involvement in treatment decisions are primary issues of patient satisfaction. One study concluded that patients did not discuss the provision of clear information as a requisite for a positive follow-up appointment experience. Instead, they highlighted their need for empathic care providers who demonstrated patience and willingness to spend time with them, through their tone of voice and body language (Molina et al.). A unified communication approach is necessary to address a patient's ability to understand and participate in improving his/her own outcomes. This approach requires healthcare providers to assess the patient's health 
literacy, level of English proficiency, and develop an understanding of the patient's cultural influences to provide patient centered care (HRSA).

Next recommendations and implications for APRNs will be discussed. 


\section{Recommendations and Implications for Advanced Nursing Practice}

Advanced practice nurses (APRNs) are poised to have an unprecedented impact on the future of our healthcare system. Whether the contribution is as a provider, through staff development, research, quality improvement or leadership, APRNs will be sought out by nurses and other members of the team.

Studies indicate that patients have difficulty understanding health information that is communicated orally during the patient-clinician interaction. Patients retain about 50\% percent of the information discussed. Studies show that those with limited health literacy are less likely to ask questions during a medical encounter, or understand medical terminology (IOM, 2004). Incorporating patient preferences into practice is challenging because of variations in patient preferences and decision making processes (Burman et al.). Lack of knowledge by patients and different healthcare beliefs may be factors in the lack of alignment between patients and clinicians (Burman et al.). More research is needed surrounding ethnic differences in communication, aspects of communication that may contribute to patient comprehension, adherence to follow-up care and patient satisfaction.

To improve patient satisfaction and adherence to follow-up care, educational programs are necessary to counsel health-care professionals with regard to language, health literacy, and empathetic communication needs (Molina et al.). Nurse leaders must ensure that nurses and other healthcare providers have the competencies necessary to ensure patient centered care (Pelletier \& Stichler).

Health professionals today are a multigenerational, diverse working group with individual psychosocial, spiritual and life experience characteristics of their own that shape their practice (Duffy). In addition they are caring for the most diverse and chronically ill patients our nation has seen. The ability to develop a compassionate, therapeutic relationship with patients and engage them as partners is critical as healthcare 
will require patients to be fully informed and active participants in self-care management (Pelletier \& Stichler).

Four critical elements have been identified as successful in aligning patient centered care and evidenced based practice: health care redesign; decision support; empowered organizational culture; and informed and empowered nurses. In addition, productive interaction between informed patients and a prepared, proactive practice team is incorporated into successful comprehensive models of care (Burman et al.). In addition, patient engagement has become interchangeable or included in the overall definition of patient centered care.

Through interpersonal aspects of professional nursing care, nurses can establish relationships that support patient engagement and activation, which can lead to improved outcomes (Pelletier \& Stichler). Evidence demonstrates that patient centered care improves disease outcomes and quality of life, and that it is critical to addressing racial, economic and social disparities in healthcare (Epstein, Fiscella, Lesser, \& Stange, 2010). Policies should assist healthcare professionals in acquiring and maintaining skills needed to support patient centered care. Organizations should strive to create a culture of patient centeredness (Epstein et al., 2010).

\section{References}

Aboumatar, H. J., Carson, K. A., Beach, M. C., Rotor, D. L., \& Cooper, L. A. (2013, November 28). The Impact of Health Literacy on Desire for Participation in Healthcare, Medical Visit communication, and Patient Reported Outcomes Among Patients with Hypertension. Journal of General Internal Medicine, 11(), 1469-76. http://dx.doi.org/10.1007/s11606-013-2466-5 
Agency for Healthcare Research and Quality (2012). Universal Precautions Tool Kit. Retrieved from http://www.ahrq.gov/professionals/quality-patient-safety/qualityresources/tools/literacy-toolkit/index.html

Burman, M. E., Robinson, B., \& Hart, A. M. (2013). Linking evidenced based practice and patient-centered care through patient preferences. Nursing Administration Quarterly, 37(3), 231-241. http://dx.doi.org/DOI:

\subsection{7/NAQ.0b013e318295ed6b}

Clear communication. (2015). Retrieved from http://www.nih.gov/institutes-nih/nihoffice-director/office-communications-public-liaison/clear-communication

Cultural Competency and Diversity. (2014). Retrieved from http://www.getceusnow.com/portal/file/culturaldiversity.htm

Duffy, J. R. (2013). Quality Caring In Nursing and Health Systems (2nd ed.). New York: Springer.

Epstein, R. M., Fiscella, K., Lesser, C. S., \& Stange, K. C. (2010, August). Why the nation needs a policy push on patient centered care. Health Affairs, 1489-1495. http://dx.doi.org/doi: 10.1377/hlthaff.2009.0888

Epstein, A. M., \& Street, R. L. (2011, March). The Values and value of patient centered care. Annals of Family Medicine, 9(2), 100-103. http://dx.doi.org/10.1370/afm.1239

Health Resources and Services Administration (HRSA), (2012). Unified Communication. Retrieved from http://www.hrsa.gov/publichealth/healthliteracy

Institute for Innovation. (2012). Retrieved from http://www.pressganey.com/searchwhole-site? indexCatalogue $=$ wholesite $\&$ searchQuery=institute for innovation $\&$ wordsMode $=0$

Institute of Medicine (2004) Health Literacy: A Prescription to End Confusion. Retrieved July 17.2014 , from 
http://www.iom.edu/ /media/Files/Report\%20Files/2004/Health-Literacy-APrescription-to-End-Confusion/healthliteracyfinal.pdf

Institute of Medicine. (2001). Crossing The Quality Chasm: A New Health System For The 21st Century [report brief]. Retrieved from www.iom.edu:

Institute of Medicine, (2010). The Future of Nursing: Leading Change, Advancing Health. Retrieved from http://www.iom.edu/reports/2010/The-Future-of-NursingLeading-Change-Advancing-Health

Komenaka, I. K., Nodora, J. N., Machado, L., Hsu, C. H., Klemens, A. E., Martinez, M. E., ... Weiss, B. D. (2014, March). Health literacy assessment and patient satisfaction in surgical practice. Surgery, 155(3) (374-83), 374-83. http://dx.doi.org/10.1016/j.surg2013.10.011

Maat, S. T. (2013, May 27). Doctors increasingly shut out of hospitals' patient experience efforts. amednews.com. Retrieved from http://www.amednews.com/article/20130527/business/130529966/4/

Molina, Y., Hohl, S. D., Ko, L. K., Rodriguez, E. A., Thompson, B., \& Beresford, S. A. (2014, April 22). Understanding the Patient -Provider Communication needs and experiences of Latina and Non-Latina White Women Following an Abnormal Mammogram. Journal of Cancer Education. Retrieved from http://www.ncbi.nlm.nih.gov/pubmed/

Pelletier, L. R., \& Stichler, J. F. (2014, September). Patient-centered care and engagement. The Journal of Nursing Administration, 44(9), 473-480. http://dx.doi.org/DOI: 10.1097/NNA.0000000000000102

Rodek, S. (2012). 4 Strategies to Boost Hospitals' HCAPS Scores. Retrieved from http://www.beckershospitalreview.com/quality/4-strategies-to-boost-hospitalshcahps-scores.html 
Rogers, G., \& Smith, D. P. (1999). Reporting comparative results from hospital patient surveys. International Journal for Quality in Healthcare, 11(), 251-9. Retrieved from http://www.ncbi.nlm.nih.gov/pubmed/10435847

Torcson, P. J. (2005). Patient satisfaction: the Hospitalist's role. Retrieved from http://www.thehospitalist.org/details/article/256805/Patient_Satisfaction_the_Hospitalists_Role.h tml

White, M., Garbez, R., Carroll, M., Brinker, E., \& Howie-Esquivel, J. (2013, MarchApril). Is "teach-back" associated with knowledge retention and hospital readmission in hospitalized heart failure patients? Journal of Cardiovascular Nursing , 28(2) (137-46), 137-46. http://dx.doi.org/10.109/ j c n.0b013e31824987bd 


\section{Appendix A}

Evaluate the following statements.

Effective Communication 100 feedback

\begin{tabular}{|c|c|c|c|}
\hline Strongly Disagree & Disagree & Neither Disagree & Agree \\
\hline
\end{tabular}

The Effective

Communication 100

modules are user friendly.

The modules contained

$\mathbf{x}$

$\mathbf{x}$

x

X

X $x$

x


The content of the

modules

is applicable to my

practice.

I would recommend the $\mathbf{x}$

100 course

\section{Effective Communication}

X

$\mathbf{X}$

$\mathbf{X}$

$\mathbf{x}$ 